\title{
Grazing-incidence diffraction from multilayers
}

\author{
G. T. Baumbach \\ Institut Laue-Langevin, Bôte Postale 156X, F38042 Grenoble Cedex 9, France
}

S. Tixier

European Synchrotron Radiation Facility, Bôtte Postale 220, F38042 Grenoble Cedex 9, France

U. Pietsch

Institut für Festkörperphysik der Universität Potsdam, 14415 Potsdam, Germany

V. Holý

Department of Solid State Physics, Faculty of Science, Masaryk University, Kotlárská 2, 61137 Brno, Czech Republic

(Received 20 October 1994)

\begin{abstract}
Grazing-incidence diffraction from superlattices has been described by a distorted-wave Born approximation. Using the example of a GaAs/AlAs superlattice, the influence of specular interface reflection on the diffraction pattern has been investigated experimentally and well explained by the theoretical treatment. A comparison with the dynamical theory and parallels with the x-ray reflectivity is given. Realstructure effects such as misorientation, interface roughness, or graduated heterotransitions have also been taken into consideration.
\end{abstract}

\section{INTRODUCTION}

Grazing-incidence diffraction (GID) is a relatively young $x$-ray scattering technique developed in the last 15 years. ${ }^{1}$ Originally applied to the investigation of nearsurface regions and perfect crystals, GID has been extended recently to investigate multilayers (ML's) and superlattices (SL). Two theoretical approachs have been employed successfully. The first is based on a distortedwave Born approximation (DWBA), considering the diffraction of a distorted wave, which penetrates the subsurface region after being transmitted through the sample surface under grazing incidence. ${ }^{2}$ The second treatment is based on the solution of the Maxwell equations for the two-beam case, solving the boundary conditions for the electrical field at the sample surface..$^{3-7}$ Mainly the first, semikinematical treatment has found practical importance since it allows one to present the diffraction intensity by an expression proportional to the kinematical scattering amplitude and thus the Fourier transformation of the diffraction potential. The application of the classical DWBA to multilayers is restricted to SL with no change in the averaged electron density, neglecting reflection at the interfaces.

The dynamical theory has been used for perfect crystals and single and double heterostructures. ${ }^{3-8}$ Numerical problems of dynamical calculations existing for more complicated multilayers and superlattices have been solved recently. ${ }^{9}$

In the case of refraction-index-modulated superlattices, additional so-called Bragg-like peaks have been detected in the scattering patterns of nonspecular reflection (NSXR) and GID. It was possible to associate these with specular interface reflection and to interpret them employing the concept of Umweganregung (excitation of a diffraction process by another diffraction). ${ }^{10,11}$

Within this paper we expand the principle of the DWBA to diffraction from multilayers and superlattices. The validity of the treatment and its advantages and limitations will be discussed. The paper will be structured as follows: Starting with the derivation of the theoretical treatment we give an interpretation of the diffraction patterns employing the concept of Umweganregung. Section III provides experimental results for GID from a superlattice. In Sec. IV we make use of the theory's ability to explain details of the diffraction pattern analytically. The fit of the experimental data allows a structural characterization with high precision including such real structure effects as misorientation and interface roughness. The discussion finishes by noting similarities between the scattering patterns of NSXR and GID.

\section{THEORETICAL TREATMENT}

The total wave field $\Psi$ after scattering of an incident wave $\Phi$ by an optical potential $V$ is fully described by the integral equation

$$
\Psi(\mathbf{r})=\Phi(\mathbf{r})+\int d \mathbf{r}^{\prime} G_{0}\left(\mathbf{r}, \mathbf{r}^{\prime}\right) V\left(\mathbf{r}^{\prime}\right) \Psi\left(\mathbf{r}^{\prime}\right),
$$

giving the complete solution of the wave equation

$$
\left(\Delta+K^{2}\right) \Psi(\mathbf{r})=V(r) \Psi(\mathbf{r}) .
$$

Here $K$ is the vacuum wave vector, and $G_{0}$ the freeparticle Green function. The optical potential $V$ is described by the space-dependent refraction index $V=K^{2}$ $[1-n(r)]$. For neutrons and $\mathrm{x}$ rays the deviation of the refraction index $n$ from 1 is in the order of $10^{-5}$. Thus $V$ can be expressed by the electrical susceptibility, $V=-K^{2} \chi(r)$ in the case of $\mathrm{x}$ rays; for neutrons $\chi(r)$ has to be replaced by the neutron scattering length $b(r)$. 
Assuming an epitaxial multilayer, we can develop the potential in a pseudo-Fourier series

$$
\chi_{\mathrm{ML}}(\mathbf{r})=\sum_{H} \chi_{H}(z) e^{-i\left\{\int_{0}^{z} H_{z}\left(z^{\prime}\right) d z^{\prime}+\mathbf{H}_{\|} \cdot \mathbf{r}_{\|}\right\}} .
$$

The Fourier coefficients of the susceptibility $\chi_{H}$ and the $z$ component of the reciprocal-lattice vectors $H$ are functions of depth.

Applying the two-beam approximation, only the Fourier components $O, H$, and $\bar{H}$ have to be taken into account.

The solution of Eq. (1) is then identical to the extended dynamical theory. It has been developed for coplanar strong asymmetric diffraction from perfect crystals and single layers on a substrate,$^{12-14}$ and for conventional GID (diffracting planes nearly perpendicular to the surface) in a series of papers. ${ }^{3-7}$ The formalism has been extended to multilayers in Ref. 8. However, numerical problems restrict the application to structures consisting of only a few layers.

Apart from the dynamical theory a number of papers are based on a second approach, employing a distortedwave Born approximation. ${ }^{15-19}$ The DWBA includes an approximation which requires the diffraction to be relatively weak. Then it can be treated by kinematical diffraction of the wave transmitted by the sample surface. The theory has been described in detail in Ref. 2 for the case of a single crystal. Up to a certain point it also gave acceptable results for multilayers with nearly no difference in the main refraction index..$^{16-19}$

\section{A. The DWBA}

In this section we want to extend the approach of the DWBA to the general case of GID from multilayers.

The essential features of the DWBA consists of splitting $V$ into two parts $V=V_{1}+V_{2}$. Using the Heisenberg representation of quantum mechanics we can develop the solution of the wave equation in the complete orthogonal basis of solutions for the wave equation

$$
\left(\Delta+K^{2}\right) \Psi^{(0)}(\mathbf{r})=V_{1}(\mathbf{r}) \Psi^{(0)}(\mathbf{r}) .
$$

$\Psi^{(0)}$ is the distorted wave field after scattering by the potential $V_{1}$. It represents the initial state for the scattering by the potential $V_{2}=V-V_{1}{ }^{20}$ Expanding the total wave field in a perturbation series and cutting after the second term, we obtain the solution of the DWBA to first order

$$
\Psi^{(1)}(\mathbf{r})=\Psi^{(0)}(\mathbf{r})+\int d \mathbf{r}^{\prime} G_{1}\left(\mathbf{r}, \mathbf{r}^{\prime}\right) V_{2}\left(\mathbf{r}^{\prime}\right) \Psi^{(0)}\left(\mathbf{r}^{\prime}\right) .
$$

Here $G_{1}$ is the Green function for a particle under action of $V_{1}$.

In order to consider multiple reflection by the interfaces, we choose

$$
V_{1}=-K^{2} \chi_{0}(r)
$$

and

$$
V_{2}=-K^{2} \chi_{H}(z) \exp \left[-i\left(\int_{0}^{z} H_{z}\left(z^{\prime}\right) d z^{\prime}+\mathbf{H}_{\|} \cdot \mathbf{r}_{\|}\right]\right] .
$$

Consequently the diffraction potential is treated as the perturbation.

As will be shown below, diffraction-generated extinction plays a role only in the very near vicinity of the fulfilled Bragg condition of the layer lattices, and is thus negligible in most parts of the multilayer truncation rod.

Applying the DWBA in this form, we consider specular reflection from the surface and from interfaces of the multilayer, and its influence on the diffracted amplitudes dynamically. However, we neglect any influence of the diffracted on the specular reflected intensities. The mutual interaction between the diffracted and reflected wave fields would require one to extend the DWBA to a higher order.

In first-order DWBA we can divide the total wave field into reflected and diffracted wave fields:

$$
\Psi^{(1)}(\mathbf{r})=\psi_{\mathrm{SXR}}(\mathbf{r})+\psi_{\mathrm{GID}}(\mathbf{r}),
$$

where the latter corresponds to the second term of Eq. (4).

The dynamical treatment of specular reflection gives the well-known nonperturbed states

$$
\begin{aligned}
& \psi^{(0)}(\mathbf{r})=e^{-i \mathbf{k}_{\|} \cdot \mathbf{r}_{\|}}\left[\begin{array}{l}
\rho(z) \\
\theta(z)
\end{array}\right], \\
& \overline{\psi^{(0)}}(\mathbf{r})=e^{-i \mathbf{k}_{\|} \cdot \mathbf{r}_{\|}}\left[\frac{\overline{\rho(z)}}{\overline{\theta(z)}}\right],
\end{aligned}
$$

where $\overline{\psi^{(0)}}$ is the irregular solution for an incident wave coming from the inside.

The reflected and transmitted wave amplitudes $\rho$ and $\theta$ can be calculated by applying the optical matrix formalism $^{21}$

$$
\left[\begin{array}{l}
\rho(z) \\
\theta(z)
\end{array}\right]=\hat{M}\left(z, z_{0}\right)\left[\begin{array}{l}
\rho\left(z_{0}\right) \\
\theta\left(z_{0}\right)
\end{array}\right]
$$

with

$$
\hat{M}=\frac{1}{T\left(z, z_{0}\right)}\left[\begin{array}{cc}
A\left(z, z_{0}\right) & R\left(z, z_{0}\right) \\
-\bar{R}\left(z_{0}, z\right) & 1
\end{array}\right]
$$

and $A\left(z, z_{0}\right)=T\left(z, z_{0}\right) \bar{T}\left(z_{0}, z\right)-\bar{R}\left(z_{0}, z\right) R\left(z, z_{0}\right)$, written for $z<z_{0}$.

$T\left(z, z_{0}\right)$ and $R\left(z, z_{0}\right)$ are the transmission and reflection functions of the layered stack between $z$ and $z_{0}$. The bar marks the functions for negative angles of incidence.

\section{B. The diffracted wave field}

The contribution of $V_{2}$ to the total wave field $\Psi^{(1)}$ at a point in space $\left(r_{\|}, z\right)$ consists of contributions from the upper part $z^{\prime}<z$ and one from the lower part $z^{\prime}>z$ of the multilayer, ${ }^{20}$

$$
\psi_{\mathrm{GID}}(r)=\psi_{<}^{(1)}(r)+\psi_{>}^{(1)}(r) .
$$

Using the two-dimensional spectral representation of the Green function, ${ }^{22}$ the diffracted wave amplitude can be expressed in the form 


$$
\psi_{\mathrm{GID}}(\mathbf{r})=\frac{1}{4 \pi^{2}} \int_{k_{\|}<K} d \mathbf{k}_{\|} \frac{e^{-i \mathbf{k}_{\|} \cdot \mathbf{r}}}{W_{k_{\|}}} \int_{-\infty}^{\infty} d r_{\|}^{\prime} \int_{0}^{\infty} d z^{\prime} e^{i \mathbf{k}_{\|} \cdot \mathbf{r}_{\|}^{\prime}}\left[\begin{array}{l}
\bar{\rho} \\
\bar{\theta}
\end{array} k_{k_{\|}}\left(z^{<}\right)\left(\begin{array}{l}
\rho \\
\theta
\end{array}\right]_{\|}\left(z^{>}\right) V_{2}\left(\mathbf{r}^{\prime}\right) \psi_{r^{\prime}}^{(0)},\right.
$$

with

$$
z<\left\{\begin{array}{l}
z^{\prime} \text { for } z>z^{\prime} \\
z \text { for } z<z^{\prime}:
\end{array}\right.
$$

The Wronskian determinant in our case is $W_{k_{\|}}=-2 i k_{z}(z)[\theta(z) \bar{\theta}(z)-\rho(z) \bar{\rho}(z)]$, which for $z<0$ becomes the easy form $W_{k_{\|}}=-2 i K_{z} \bar{\theta}(-0)$. After substituting Eq. (5), integration over $r_{\|}$gives

$$
\psi_{\mathrm{GID}}(r)=\int_{k_{\|}<K} d \mathbf{k}_{\|} \frac{e^{-i \mathbf{k}_{\|} \cdot \mathbf{r}}}{W_{k_{\|}}} \delta\left(\mathbf{k}_{\|}-\mathbf{k}_{0 \|}-\mathbf{H}_{\|}\right) \int_{0}^{\infty} d z^{\prime}\left(\begin{array} { l } 
{ \overline { \rho } } \\
{ \overline { \theta } }
\end{array} \mathbf { k } _ { \| } ( z ^ { < } ) \left(\begin{array}{l}
\rho \\
\theta
\end{array} \mathbf{k}_{\|}\left(z^{>}\right) \chi_{H}\left(z^{\prime}\right) e^{-i\left\{\int_{0}^{z^{\prime}} H_{z}\left(z^{\prime \prime}\right) d z^{\prime \prime}\right\}}\left[\begin{array}{l}
\rho \\
\theta
\end{array}\right]_{0}\left(z^{\prime}\right),\right.\right.
$$

and thus establishes the in-plane Bragg condition

$$
\mathbf{k}_{h \|}=\mathbf{k}_{0 \|}+\mathbf{H}_{\|}
$$

to be fulfilled everywhere in the crystal. In other words, it provides the crystal truncation rod of the multilayer.

We are interested in the wave amplitude at the position of our detector at $z<0$. The amplitude of $\psi_{\mathrm{GID}}$ can be expressed as a sum over contributions of the substrate (indexed here as layer 0 ) and the different layers in the structure:

$\psi_{\mathrm{GID}}(z<0)=\frac{i}{2} K^{2} \frac{e^{-i \overline{\mathbf{K}}_{h} \cdot \mathbf{r}}}{K_{h z}} \sum_{i}\left(\rho_{h}, \theta_{h}\right)_{i} S_{i}^{h}\left(\begin{array}{l}\rho_{0} \\ \theta_{0}\end{array}\right)_{i}$

with

$$
\widehat{S_{i}^{h}}=\int_{z_{i}}^{z_{j}} d z^{\prime} \chi_{H i}\left[\begin{array}{ll}
e^{-i q_{11^{z}}^{i}} & e^{-i q_{12^{z}}^{i}} \\
e^{-i q_{21}^{i} z} & e^{-i q_{22^{z}}^{i}}
\end{array}\right]
$$

Here $\rho_{i}$ and $\theta_{i}$ are the amplitudes of the reflected and transmitted waves at the upper inner interface of the layer $i$. The index 0 is the initial state, and $h$ the final state after diffraction. Provided the lateral Bragg condition is exactly fulfilled, there occur four scattering processes in each layer. These are characterized by reduced scattering vectors in the layers,

$$
\begin{aligned}
& q_{11}=k_{o z}+k_{h z}-H_{z}, \quad q_{12}=k_{o z}-k_{h z}-H_{z}, \\
& q_{21}=-k_{o z}+k_{h z}-H_{z}, \quad q_{22}=-k_{o z}-k_{h z}-H_{z},
\end{aligned}
$$

corresponding to the full scattering vectors $\mathbf{Q}_{i j}$ of Fig. 1 .

For classical GID (diffraction planes perpendicular to the surface), only two scattering vectors, e.g., $q_{22}$ and $q_{12}$, are independent. The two others differ from them only in sign. Notice that all scattering vectors vary from layer to layer, since

$$
k_{0_{z}}(z)=\sqrt{\left(K^{2}-k_{0_{I I}}^{2}\right)+K^{2} \chi_{0}(z)}
$$

and

$$
k_{h_{z}}(z)=\sqrt{\left(K^{2}-k_{h_{I I}}^{2}\right)+K^{2} \chi_{0}(z)} .
$$

The scattering situation within one layer will be demon- strated schematically in Fig. 1 by the concept of Umweganregung. Measuring in reflection geometry, $q_{22}$ describes the primary scattering process. The corresponding matrix element $S_{22}$ for one layer is similar to the result of the kinematical theory. Three other processes are caused by Umweganregung. They presuppose simultaneous specular interface reflection.

To obtain the contribution of one scattering process of the whole layered stack we have to take the sum over all contributions of the layers considering the right phase relations. Considering only the specular reflection from the sample surface and neglecting the interface reflection, the reflected wave amplitude becomes zero inside the multilayer $\left[\rho^{*}(z>0)=0\right]$, and the transmitted wave has the form $\theta^{*}(z>0)=t e^{-i k_{z} Z}$ with the Fresnel coefficient of transmission of the surface $t$. Replacing $\theta$ and $\rho$ by $\theta^{*}$ and $\rho^{*}$, we get the well-known expression used conventionally for the scattered wave of GID:

$$
\begin{aligned}
\psi_{\mathrm{GID}}(z<0)= & \frac{i}{2} K^{2} \frac{e^{-i \overline{\mathrm{K}}_{h} \cdot \mathrm{r}}}{K_{h z}} t_{h} t_{0} \\
& \times \int_{0}^{\infty} d z^{\prime} \chi_{H} e^{-i \int_{0}^{z^{\prime}} q_{22} d z^{\prime \prime}},
\end{aligned}
$$

where the scattered amplitude is proportional to the Fourier transformation of the diffraction potential.

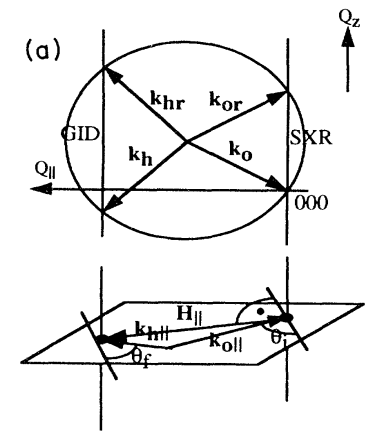

(b)

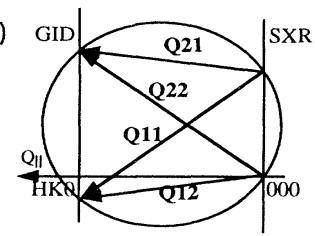

FIG 1. Schematical situation of scattering within the layers. (a) The wave vectors of the unperturbed states: the projections on the lateral $\left(Q_{x} Q_{y}\right)$ plane and on the $\left(Q_{z} H_{\|}\right)$plane. (b) The GID scattering vectors of the four processes [they lie in the $\left(Q_{z} H_{\|}\right)$plane]. 
To illustrate the difference between the primary scattering process of a multilayer in Eq. (12) and the expression of the conventional GID [Eq. (14)], in Fig. 2 we compare the transmission function $\theta$ for a AlAs/GaAs heterostructure (single layer on a substrate) with $\theta^{*}$ of a simple substrate. In Fig. 2 we have calculated $\theta^{*}$ and $\theta$ directly below the surface. Figure 2(a) shows the wellknown behavior of the transmission function through a single surface. However, we find oscillations in Fig. 2(b), related to the AlAs-layer thickness.

For a very thin AlAs layer we see, in the diffraction pattern calculated with Eq. (12), a splitting of the Yoneda-like wings, as is known from NSXR by multilayers. ${ }^{11}$

\section{Grazing-incidence diffraction from a superlattice}

Let us now consider the special case of diffraction from a superlattice. The periodic modulation of the crystalline properties gives rise to satellites in the truncation rods through reciprocal-lattice points. The superlattice periodicity allows us to transform Eq. (12). We define the matrices $\widehat{M_{a / b}}$ and $\widehat{M_{b / a}}$ to relate the specular wave field amplitudes at the $A$ - and $B$-type interfaces. The matrix $\widehat{M}_{\mathrm{sl}}\left(z, z-D_{\mathrm{sl}}\right)=\widehat{M_{a / b}} \widehat{M_{b / a}}$ relates the specular wave field from one SL period to the next. Now for Eq. (12) we write the expression

$$
\begin{aligned}
& \psi_{\mathrm{GID}}(z<0)=\frac{i}{2} K^{2} \frac{e^{-i \overline{\mathbf{K}}_{h} \cdot \mathrm{r}}}{K_{h z}} t_{h}\left\{\sum_{n=0}^{N-1}\left[\widehat{M}_{\mathrm{s} l 0}^{n}\left[\begin{array}{c}
\rho\left(+z_{\mathrm{sl}}\right) \\
\theta\left(+z_{\mathrm{sl}}\right)
\end{array}\right]\right]_{h}^{T} \widehat{S}_{\mathrm{sl}}\left[\widehat{M}_{\mathrm{s} l h}^{n}\left[\begin{array}{l}
\rho\left(+z_{\mathrm{sl}}\right) \\
\theta\left(+z_{\mathrm{sl}}\right)
\end{array}\right]\right]_{0}\right] t_{0}, \\
& \widehat{S_{\mathrm{sl}}}=\left[\widehat{M}_{\mathrm{s} 1 h} S_{a} M_{\mathrm{s} 10}+\widehat{M_{a / b h}} S_{b} \widehat{M_{a / b 0}}\right] .
\end{aligned}
$$

Here $S_{\mathrm{sl}}$ can be understood as the diffraction matrix of the superlattice period including all four multiplescattering processes. The sum runs over the number of periods $N$. $+z_{\text {sl }}$ is the position of the inner substrate surface.

\section{Numerical calculations and comparison with the dynamical theory}

The theoretical treatment has been employed to calculate the diffracted intensities for $\lambda=1.54 \AA$ of the (220) reflection for the example of a 20-period GaAs/AlAs superlattice. Each period consists of a $66.5-\AA$-thick GaAs layer and a 147.5- $\AA$-thick AlAs layer. Measurements of GID intensity are usually carried out by keeping the incident angle with respect to the surface $\alpha_{i}$ as a fixed parameter, and measuring the diffraction pattern as a function of the exit angle with respect to the sample surface $\alpha_{f}\left(\alpha_{f}\right.$ scan) [Fig. 3(a)]. In Fig. 4 the intensity pattern of an $\alpha_{f}$ scan has been calculated for $\alpha_{i}=0.76^{\circ}$. We see a large number of peaks, but only some of them coincide with the expected positions of the diffraction satellites. The curves below, Figs. 4(b)-4(e), give the square of the amount of the diffraction amplitudes calculated for the four diffraction processes separately.

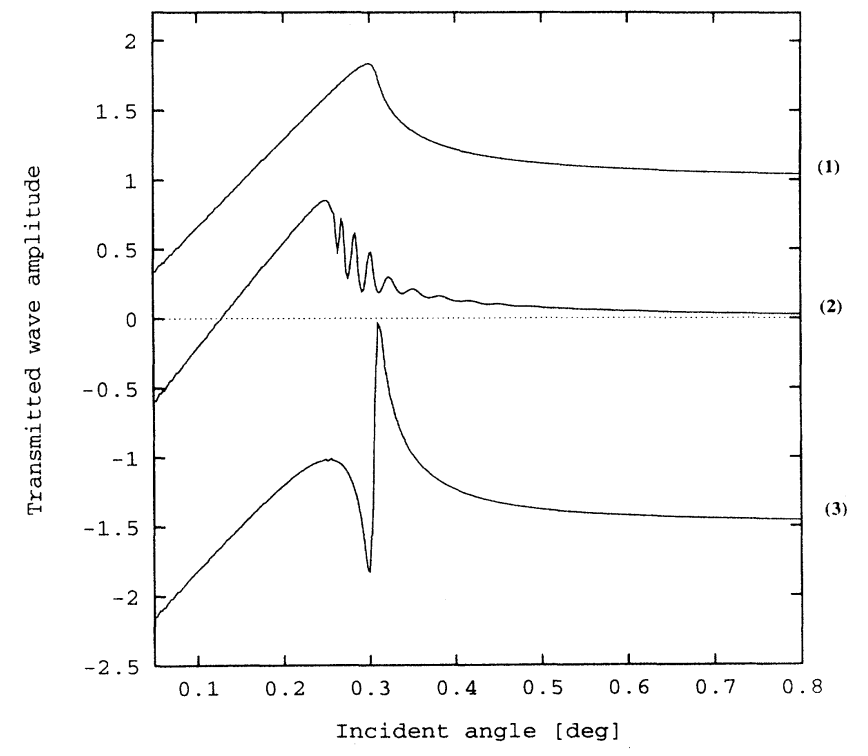

FIG. 2. Amount of the transmitted wave amplitude at the inner surface. (1) GaAs substrate. (2) 100-nm AlAs on GaAs. (3) 10-nm GaAs on AlAs (the curves are shifted progressively along the intensity scale).
The $q_{22}$ process (primary process) shows exclusively the expected true superlattice satellites. The other additional peaks can be attached to the three secondary processes.

In Fig. 5 we compare the results of the DWBA with calculations of the dynamical theory. We do not enter into detail for the numerical procedure of the dynamical simulations, since it is the subject of another paper. ${ }^{9}$

Figure 5 shows good agreement between both treatments over most of the $\alpha_{f}$ scan. Near $\alpha_{f}=\alpha_{i}$ the DWBA calculates higher intensities. There the incident beam and the diffracted beam nearly fulfill the Bragg condition for the diffraction plane $H K O$. The diffraction causes extinction that is not considered by the DWBA approach.

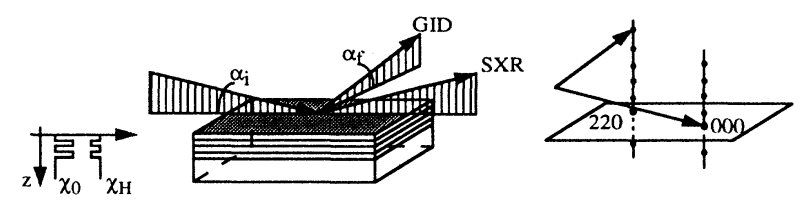

FIG. 3. Grazing-incidence diffraction by a superlattice; schematical representation in real and reciprocal space. 

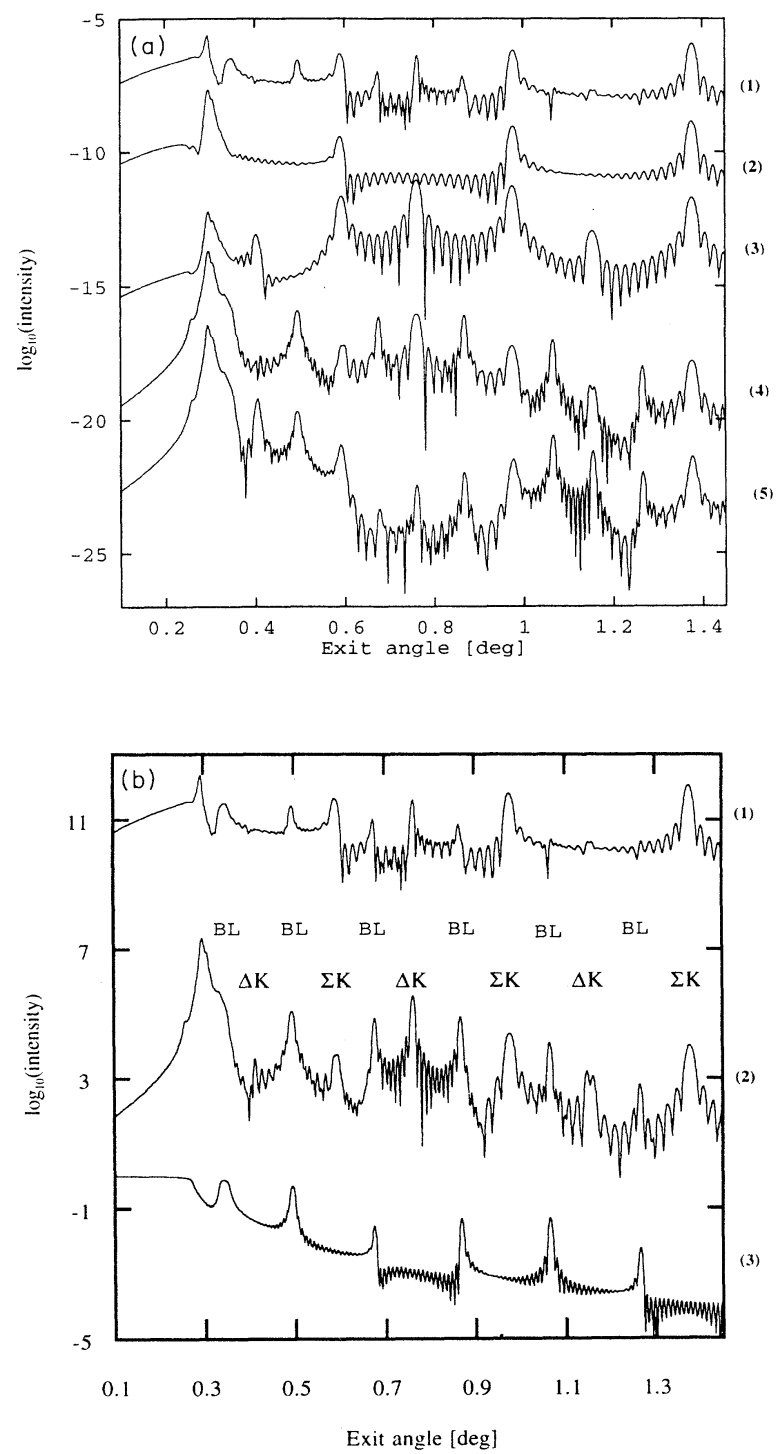

FIG. 4. Calculated (220) $\alpha_{f}$ scans of a AlAs/GaAs superlattice for $\alpha_{i}=0.76^{\circ}$. (a) Results of the four scattering processes: (1) Whole GID intensity including all four processes. (2) The primary scattering process $q_{22}$. (3) The secondary scattering process $q_{12}$. (4) The secondary scattering process $q_{21}$. (5) The secondary scattering process $q_{11}$. (b) Classification and origin of the three kinds of peaks. (1) Whole GID intensity including all four processes. (2) Contribution of the three secondary processes. (3) Specular reflection curve (the curves are shifted progressively along the intensity scale).

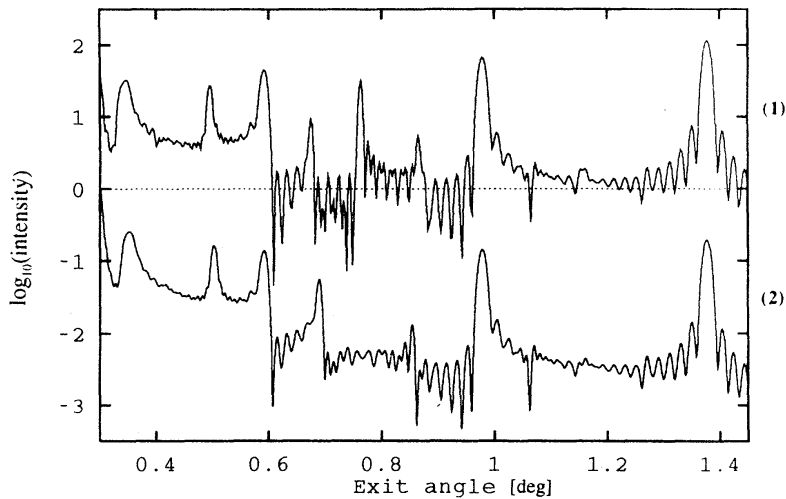

FIG. 5. Comparison of $\alpha_{f}$ scans (relative intensities) calculated by (1) the present DWBA and (2) the dynamical theory.

\section{EXPERIMENT}

Experiments to study GaAs/AlAs superlattices were carried out with the surface diffractometer D4 at HASYLAB Hamburg. The experimental setup is described in detail in Ref. 17. Here we show the results obtained from a GaAs/AlAs superlattice of 20 periods with nominal 7.0-nm-thick GaAs layers and 15.0-nm-thick A1As layers, grown on a [001]-GaAs substrate by molecular-beam epitaxy (MBE). We adjusted the lateral Bragg condition for $\alpha_{i}=0.4^{\circ}$, and measured the $\alpha_{f}$ scans by use of a one-dimensional position-sensitive detector. The angular scale was calibrated beforehand by measurements in the reflection mode. Some $\alpha_{f}$ scans for different $\alpha_{i}$ 's are selected in Fig. 6(a). Figure 6(b) gives the same curves in $Q_{z}$ coordinates. For small $\alpha_{i}$ the wave does not penetrate the whole SL, giving rise to increased full widths at half maximum (FWHM) of the satellites.

Near the critical angle appears a split Yoneda-like peak. We find the primary true satellites at nearly constant $Q_{z}$ positions. Additionally we observe several multiple-scattering peaks, as predicted by the theory. The multiple scattering peaks have fixed positions in the $\alpha_{f}$ representation [Fig. $6(\mathrm{a})$ ].

\section{DISCUSSION}

In order to explain the peak positions of the additional peaks analytically, we derivate a semikinematical approximation for the four scattering processes. In the kinematical approximation of SXR we neglect multiple reflection between the interfaces. Then $\widehat{M_{\mathrm{sl}}}$ as well as $\widehat{M_{a / b}}$ assume simple forms:

$$
\widehat{M_{b / a}}=\left[\begin{array}{cc}
e^{-i k_{z} t_{a}} & r e^{-i k_{z} t_{a}} \\
0 & e^{+i k_{z} t_{a}}
\end{array}\right] \text { and } \widehat{M}_{\mathrm{sl}}^{n}=e^{+i n k_{\mathrm{z}} D_{\mathrm{sl}}}\left[\begin{array}{cc}
e^{-i 2 n k_{z} D_{\mathrm{sl}}} & \sum_{j=0}^{n-1} F_{\mathrm{sl}} e^{-i 2 j k_{z} D_{\mathrm{sl}}} \\
0 & 1
\end{array}\right] \text {, }
$$

with the superlattice structure factor for specular reflection

$$
F_{\mathrm{sl}}=2 i r e^{-i k_{z b} t_{b}} \sin \left(k_{z a} t_{a}\right) e^{-i\left(k_{z b} t_{b}+k_{z b} t_{b}\right)} .
$$

This allows us to write analytical solutions for the amplitudes of the four scattering processes, 

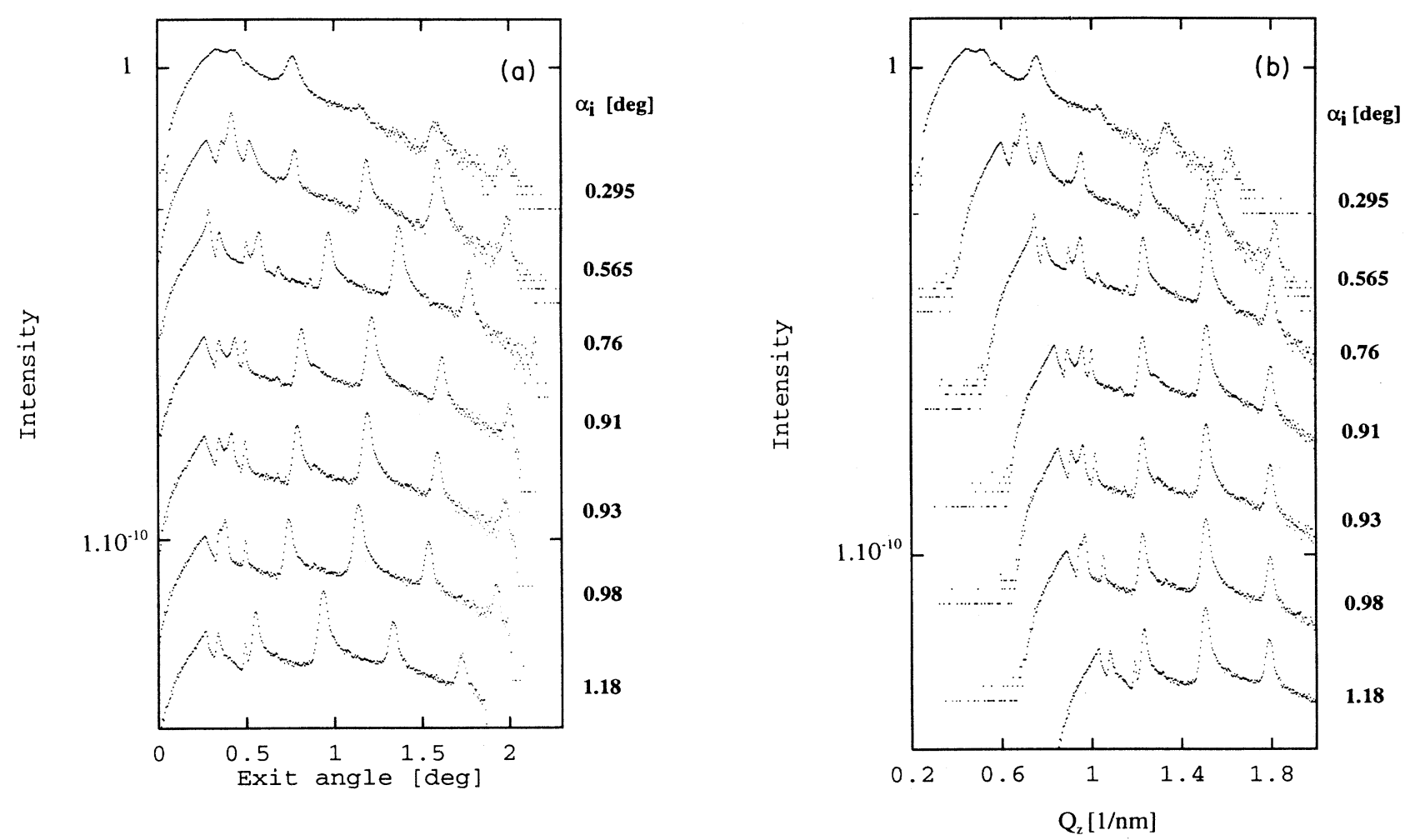

FIG. 6. Experimental GID curves of a 20-period GaAs/AlAs superlattice: (a) $\alpha_{f}$ scans and (b) in the $Q_{z}$ representation (the curves are shifted progressively along the intensity scale).

$$
\psi_{\mathrm{GID}}(z<0)=\frac{i}{2} K^{2} \frac{e^{-i \overline{\mathbf{K}}_{h} \mathrm{r}}}{K_{h z}} t_{h}\left[\begin{array}{ll}
\varphi_{11} & \varphi_{12} \\
\varphi_{21} & \varphi_{22}
\end{array}\right] t_{0},
$$

which are apart from a common phase factor:

$$
\begin{aligned}
& \varphi_{11}=\frac{S_{11} F_{\mathrm{sl}}^{0} F_{\mathrm{sl}}^{h}}{\left(e^{+i 2 k_{0 z} D_{\mathrm{sl}}}-1\right)\left(e^{+i 2 k_{0 z} D_{\mathrm{sl}}}-1\right)}\left\{\left(\frac{e^{+i \Sigma k_{z} N D_{\mathrm{sl}}}-1}{e^{+i \Sigma k_{\mathrm{z}} D_{\mathrm{sl}}}-1}-\frac{e^{+i \Delta k_{z} N D_{\mathrm{sl}}}-1}{e^{+i \Delta k_{\mathrm{z}} D_{\mathrm{sl}}}-1}\right)+\left(\frac{e^{-i \Sigma k_{z} N D_{\mathrm{sl}}}-1}{e^{-i \Sigma k_{z} D_{\mathrm{sl}}}-1}-\frac{e^{+i \Delta k_{z} N D_{\mathrm{sl}}}-1}{e^{+i \Delta k_{z} D_{\mathrm{sl}}}-1}\right)\right\}, \\
& \varphi_{12}=\frac{S_{12} F_{\mathrm{sl}}^{h}}{e^{+i 2 k_{h z} D_{\mathrm{sl}}}-1}\left(\frac{e^{+i \Delta k_{\mathrm{z}} N D_{\mathrm{sl}}}-1}{e^{+i \Delta k_{\mathrm{z}} D_{\mathrm{sl}}}-1}-\frac{e^{+i \Sigma k_{\mathrm{z}} N D_{\mathrm{sl}}}-1}{e^{+i \Sigma k_{\mathrm{z}} D_{\mathrm{sl}}}-1}\right), \\
& \varphi_{21}=\frac{S_{21} F_{\mathrm{sl}}^{0}}{e^{+i 2 k_{0 z} D_{\mathrm{sl}}}-1}\left(\frac{e^{-i \Delta k_{\mathrm{z}} N D_{\mathrm{sl}}}-1}{e^{-i \Delta k_{\mathrm{z}} D_{\mathrm{sl}}}-1}-\frac{e^{+i \Sigma k_{\mathrm{z}} N D_{\mathrm{sl}}}-1}{e^{+i \Sigma k_{\mathrm{z}} D_{\mathrm{sl}}}-1}\right), \\
& \varphi_{22}=S_{22} \frac{e^{+i \Sigma k N D_{\mathrm{sl}}}-1}{e^{+i \Sigma k D_{\mathrm{sl}}}-1} \text { with } \Sigma k_{z}=k_{h z}+k_{0 z}, \Delta k_{z}=k_{0 z}-k_{h z} .
\end{aligned}
$$

Keeping in mind that in an $\alpha_{f}$ scan $\alpha_{i}$ remains unchanged, three kinds of peaks will be created, if one of the terms between the parentheses in the denominator goes to zero:

$\Sigma k_{z} D_{\mathrm{sl}} / 2=h \pi, \quad \Delta k_{z} D_{\mathrm{sl}} / 2=l \pi, \quad k_{h z} D_{\mathrm{sl}}=n \pi$.

Thus we can classify four different types of features in the complete truncation rods: first we have the Yoneda-like wings, which are situated at the critical angle for total external reflection $\theta_{c}$. In the case of thin subsurface layers there occurs what we know as frustrated total reflection from the optics of thin films. That is, the transmitted evanescent wave reaches the next interface. It leads to a splitting of the Yoneda-like wings caused by 
the behavior of the transmitted wave amplitude $\theta$, as we have seen in Fig. 2. The primary true diffraction satellites occur if the sum of the inner wave vectors $\Sigma k_{z}=k_{0 z}+k_{h z}$ is equal to a reciprocal superlattice vector.

Two types of Umweganregung peaks are generated in the $\alpha_{f}$ scans by the processes $\varphi_{11}, \varphi_{12}$, and $\varphi_{21}$. First there occur peaks whenever the exit wave fulfills the Bragg condition of the superlattice; that is, two times in the angular space between two neighboring true satellites.

Such peaks are known from the diffuse scattered intensities of NSXR, here called Bragg-like resonances. A further type of peak arises from the terms containing the difference of wave vectors $\Delta k_{z}$. In Fig. 4(b) the different kind of peaks are marked in an $\alpha_{f}$ scan. For an illustration of the origin of the different peaks, we added the specular reflection curve and the sum of the three Umweganregung processes in Fig. 4(b).

If $\alpha_{i}$ fulfills the Bragg condition of specular reflection, the positions of the $\Delta k_{z}$ satellites fall together with the true $\Sigma k_{z}$ satellites and with every second Bragg-like resonance (Fig. 7). Adding in Eq. (15), the amplitudes of the multiple-scattering processes phase relations play a role. Thus Umweganregung can violate rules for forbidden satellite orders and, conversely, destructive interference can give rise to diminished true satellites (see Fig. 8).

\section{A. The $\left(Q_{z}, \alpha_{i}-\alpha_{f}\right)$ mapping}

Generally, it follows from symmetry considerations that in a noncoplanar scattering experiment the measured intensity is not, as in the coplanar case, completely deter-

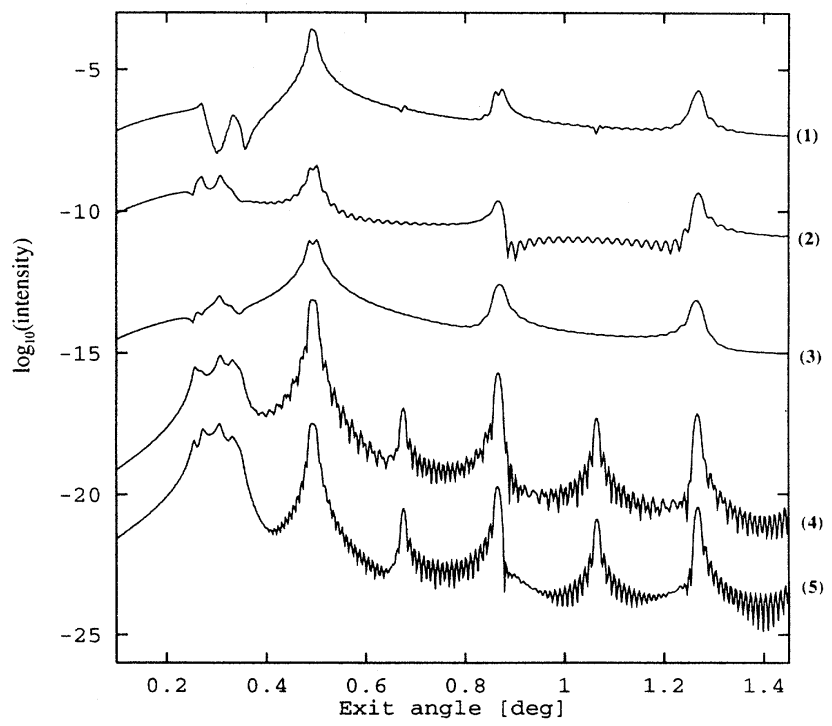

FIG. 7. Calculated (220) $\alpha_{f}$ scans for $\alpha_{i}=0.49^{\circ}$ (fulfilled Bragg condition for the second SXR Bragg peak). (1) Whole GID intensity including all four processes. (2) The primary scattering process $q_{22}$. (3) The secondary scattering process $q_{12}$. (4) The secondary scattering process $q_{21}$. (5) The secondary scattering process $q_{11}$ (the curves are shifted progressively along the intensity scale).

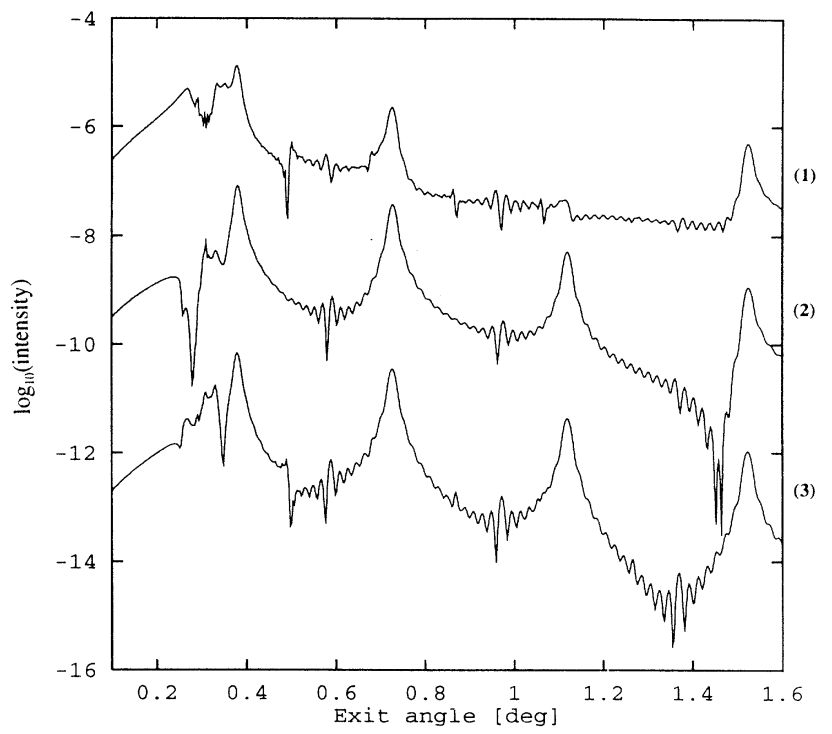

FIG. 8. Calculated (220) $\alpha_{f}$ scans for $\alpha_{i}=0.30^{\circ}$. Destructive interference between the primary and secondary processes causes the attenuation of a true satellite: (1) whole GID intensity; (2) the primary scattering process; and (3) the secondary processes (the curves are shifted progressively along the intensity scale).

mined by the vacuum wave-vector length $K$ and the scattering vector $\mathrm{Q}, I(K, \mathrm{Q})$. The scattering amplitude depends on $\mathbf{k}_{0}$ and $\mathbf{k}_{h}$ rather than only on their difference vector $\mathbf{Q}$.

In addition to the wave vector in vacuum $K$, we need at least four independent coordinates, which, e.g., in angular space can be the angles $\alpha_{i}, \alpha_{f}, \Theta_{i}$, and $\Theta_{f}$ [see Fig. 1(a)].

However, as is shown in Eq. (10), all calculations of the diffraction pattern along the truncation rod predict a fulfilled lateral Bragg condition within the sample. $k_{h \|}=k_{0 \|}+H_{\|}$.

Taking the lateral Bragg condition into account (which means in a perfect multilayer we measure only the nonzero intensity along the truncation rods), $\Theta_{i}$ and $\theta_{f}$ become functions of $\alpha_{i}, \alpha_{f}$, and $H_{\|}$(Fig. 9):

$$
\begin{aligned}
& \sin \left(\Theta_{i}\right)=\frac{K_{0 z}^{2}-H_{\|}^{2}-K_{h z}^{2}}{-2 H_{\|} \sqrt{K^{2}-K_{0 z}^{2}}}, \\
& \sin \left(\Theta_{f}\right)=\frac{-K_{0 z}^{2}-H_{\|}^{2}+K_{h z}^{2}}{-2 H_{\|} \sqrt{K^{2}-K_{h z}^{2}}},
\end{aligned}
$$

with

$$
K=\frac{2 \pi}{\lambda}, \quad K_{0 z}=\frac{2 \pi}{\lambda} \sin \left(\alpha_{i}\right), \quad K_{h z}=\frac{2 \pi}{\lambda} \sin \left(\alpha_{f}\right) .
$$

Assuming the experimental situation fulfils Eq. (19), the GID intensity of a perfect epitaxial multilayer can b $\approx$ described only by $\alpha_{i}$ and $\alpha_{f}$.

Drawing the diffraction patterns of the truncation rod as functions of $\alpha_{i}$ and $\alpha_{f}$, we find the profile of Fig. 10. 

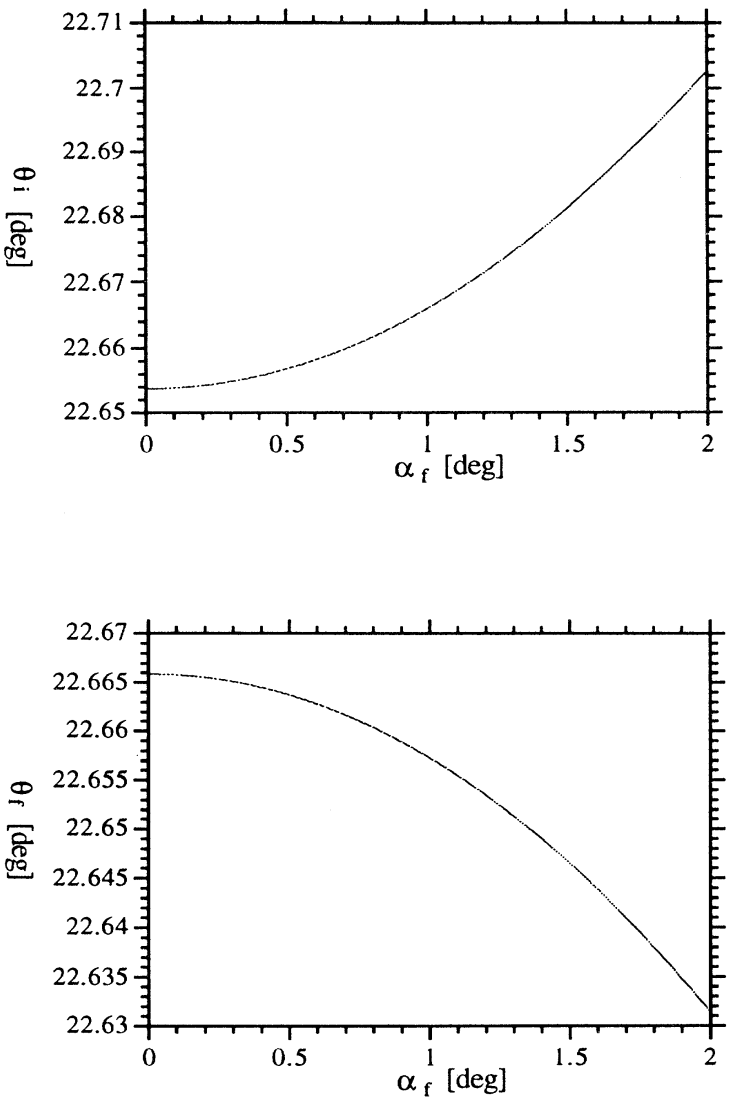

FIG. 9. $\theta_{i}$ and $\theta_{f}$ as functions of $\alpha_{f}$ for $\alpha_{i}=0.76^{\circ}$ and the fulfilled in-plane (220) Bragg condition.

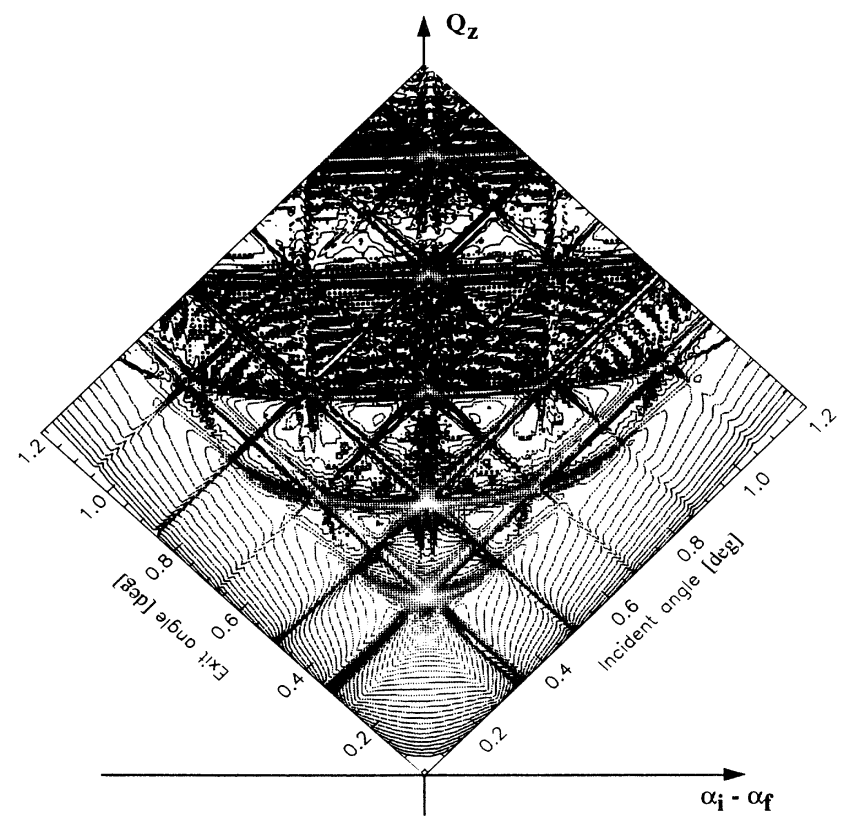

FIG. 10. Contour plot of a calculated $\left(Q_{z}, \alpha_{i}-\alpha_{f}\right)$ mapping; (220) GID intensity of the GaAs/AlAs superlattice.
The area accessible in reflection geometry is limited by the sample horizon, represented by the lines $\alpha_{i}$ and $\alpha_{f}=0$. The position and classification of the different kinds of diffraction peaks is illustrated in the schematical representations of Fig. 11, neglecting [Fig. 11(a)] and including [Fig. 11(b)] the mean refraction in the superlattice. The constant $\alpha_{i}$ and $\alpha_{f}$ lines, where the Bragg condition for the superlattice Bragg peaks of the reflection mode are fulfilled, form a grid of Bragg-like resonance lines in the $\left(Q_{z}, \alpha_{i}-\alpha_{f}\right)$ mapping. Their grid points, the so-called Bragg-like peaks, can be determined by the two
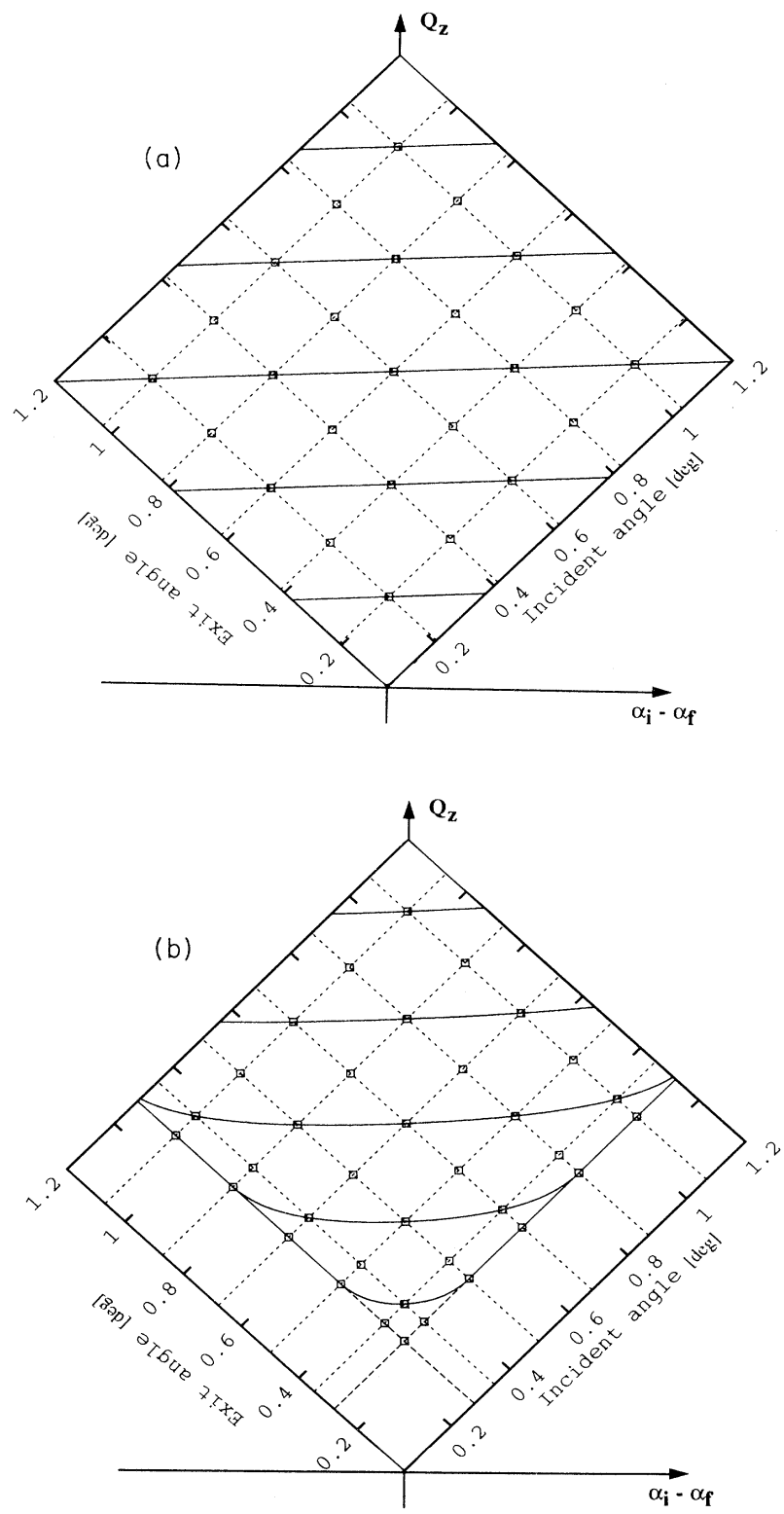

FIG. 11. Positions of the primary true satellites (plain lines), the Bragg-like resonance lines (dashed lines), and Bragg-like peaks (square dots) in the $\left(Q_{z}, \alpha_{i}-\alpha_{f}\right)$ mapping (a) without refraction and (b) including refraction. 
integers $n$ and $m$ according to the modified Bragg equations

$$
2 D_{\mathrm{sl}} \sqrt{\sin ^{2} \alpha_{i}+\left\langle\chi_{0}\right\rangle}=n \lambda
$$

and

$$
2 D_{\mathrm{sl}} \sqrt{\sin ^{2} \alpha_{f}+\left\langle\chi_{0}\right\rangle}=m \lambda,
$$

from which we get the $Q_{z}$ positions

$Q_{n m}=\left[\frac{n^{2} \pi^{2}}{D_{\mathrm{sl}}}-K^{2} \chi_{0}(z)\right]^{1 / 2}+\left(\frac{m^{2} \pi^{2}}{D_{\mathrm{sl}}}-K^{2} \chi_{0}(z)\right]^{1 / 2}$.

The primary true satellites are at constant $q_{z}$ lines in Fig. 11(a). They become bent due to refraction and flow asymptotically into the lines $\alpha_{i}=\theta_{c}$ and $\alpha_{f}=\theta_{c}$ of the Yoneda-like wings in Fig. 11(b). Going back to the intensity mapping of Fig. 10, we find the true satellites forming banana-shaped features in the $\left(Q_{z}, \alpha_{i}-\alpha_{f}\right)$ space following the Bragg condition

$\sin \alpha_{f}=\left\{\left\{\frac{h 2 \pi / D_{\mathrm{sl}}+H_{z}+K \sqrt{\sin ^{2} \alpha_{i}+\chi_{0 \mathrm{sl}}}}{-K}\right\}^{2}-\chi_{0 \mathrm{sl}}\right]^{1 / 2}$.

For $H_{z}=0$ the banana-shaped features cross the Bragglike resonance lines within the Bragg-like peaks. The $\Delta K$ satellites are lines parallel to $Q_{z}$, but they are of low intensity.

The measured $\alpha_{f}$ scans correspond to sections of constant $\alpha_{i}$. One calculated section along the Bragg-like resonance line was given in Fig. 7 and discussed above. Another section between two resonance lines corresponds to Fig. 4.

\section{B. Real-structure influences}

Without considering real-structure effects it is approximately possible to confirm the positions of the experimental peaks by simulation, but there remain problems in fitting the intensity ratios of the satellites for a nonperfect sample.

In this section we study the influence of a slight misorientation, of sample roughness, and of graduated heterotransitions on the diffraction pattern, and we demonstrate the sensitivity of the diffraction method for real structure characterization.

\section{Misorientation}

In the case of growth on a misoriented substrate or of orthorhombic or monoclinic distortion due to lattice mismatch of the layers, the reciprocal-lattice vector contains a nonzero $z$ component $H_{z}$. Then in Eq. (13) we obtain four different magnitudes of $q$. This affects the peak positions of the four scattering processes. Here let us consider, for simplicity, a small- $H_{z}$ constant over depth (an identical misorientation for all layers, but conserved tetragonal distortion). The slight misorientation gives rise to a constant shift of all primary satellites in the $Q_{z}$

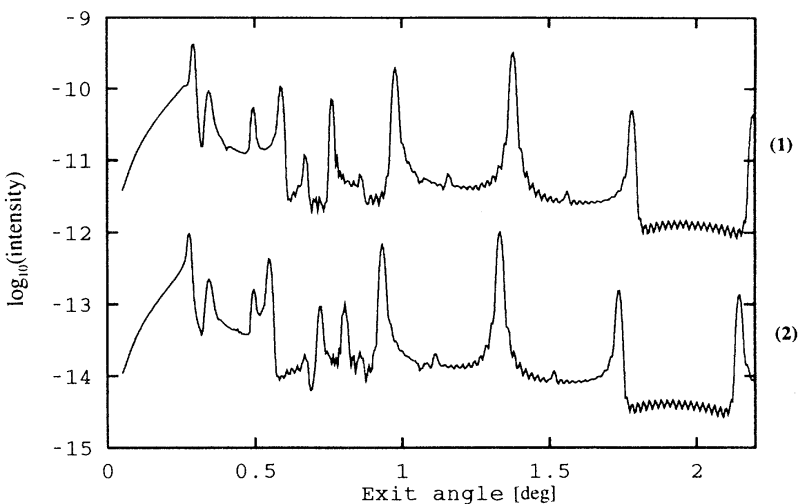

FIG. 12. Influence of misorientation on the (220) $\alpha_{f}$ scans (relative intensities) for $\alpha_{i}=0.76^{\circ}$, no misorientation (1), and $0.06^{\circ}$ misorientation (2).

representation of Fig. 12, where the Bragg-like peaks are not affected. Second, $q_{12}+q_{21}$ is no longer zero but two times $H_{z}$. That causes a splitting of the $\Delta K$ lines in the $\left(Q_{z}, \alpha_{i}-\alpha_{f}\right)$ mapping. Further, the banana-shaped features no longer cross the Bragg-like lines at the exact positions of the Bragg-like peaks of Umweganregung (Fig. 13).

Figure 12 illustrates the sensitivity of the intensity ratio and the positions of the Bragg-like peaks compared with the true satellites with respect to a very small variation of $H_{z}$. By fitting the exact positions, we found a misorientation of the superlattice (220) planes of $0.03^{\circ}$.

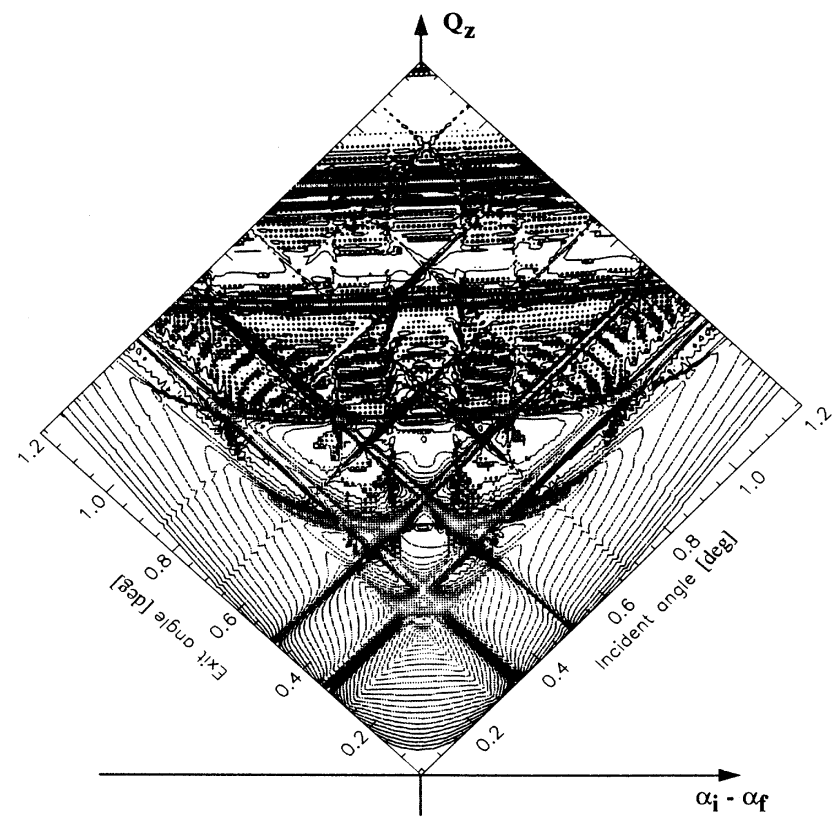

FIG. 13. Contour plot of a calculated real-structure $\left(Q_{Z}, \alpha_{i}-\alpha_{f}\right)$ mapping with a $0.15^{\circ}$ misorientation and constant rms roughness of all interfaces $(\sigma=1.5 \mathrm{~nm})$. 


\section{Graduated heterotransitions and interface roughness}

Interface defects in multilayers such as interface roughness, graduated heterotransitions, or interdiffusion have been investigated by conventional coplanar $x$-ray diffraction and $x$-ray reflection by many authors. Here we want to describe the influence on the coherent GID truncation rods.

Unlike to heterotransitions, rough interfaces generate diffuse scattering. The diffuse scattering of superlattices in x-ray reflection and in the GID has been the subject of another paper. ${ }^{23}$ In the direction of the coherent wave it is difficult to distinguish between roughness and graduated interfaces. A rough interface interacts there like a composition transition layer.

Thus we can consider the influence of rms roughness on the coherent crystal truncation rod to be similar to the treatment of a graduated heterotransition.

First, the graduated interface affects the nonperturbed states; that is, the specular reflected wave field. Treating the heterotransition dynamically, for the amplitude ratio of the transmitted and reflected waves we found the differential equations ${ }^{24}$

$$
\frac{d X}{d z}=\frac{d r}{d z}\left(1-X^{2}\right)-2 i K_{z} X
$$

with

$$
X=\rho / \theta \text { and } \frac{d r}{d z}=\frac{1}{\sin \left(2 \alpha^{\prime}\right)} \frac{d \alpha^{\prime}}{d z} .
$$

$\alpha^{\prime}$ is the $z$-dependent angle of refraction. Notice the analogy with the Takagi-Taupin equation of diffraction. In our case of very thin transition layers, the quadratic term in Eq. (23) can be neglected within the graduated interface (the semikinematical treatment). For the special case of a Gaussian profile the semikinematical treatment of the single heterotransition leads to the formulas of Nevot and Croce for the reflection coefficients. ${ }^{25}$

The influence of the interface grading in the perturbation potential is similar to that in conventional $x$-ray diffraction. The strong diffraction orders will be diminished with increasing transition width, and rules for forbidden satellites can be broken.

For identical superlattice periods (constant roughness) it makes sense to calculate first $F_{\mathrm{sl}}, S_{\mathrm{sl}}$, and $M_{\mathrm{sl}}$ for one period and to prefer the optical matrix formalism compared with a recursion formalism. A more detailed description is given in Ref. 24. In Fig. 14 the calculated curves for different rms roughnesses (assuming a Gaussian profile) show that mainly the Bragg-like peaks diminish, since the reflectivity of the interfaces is reduced. Thus in the real-structure mapping of Fig. 13 the intensity of the Bragg-like resonance lines decreases with increasing $\alpha_{i}$ and $\alpha_{f}$. The banana-shaped features of true satellites are less affected. However, we have found that along the Bragg-like resonances, the intensity at the position of true satellites can also vary dramatically due to graduated interfaces, since there the banana-shaped features fall together with the Bragg-like peaks.

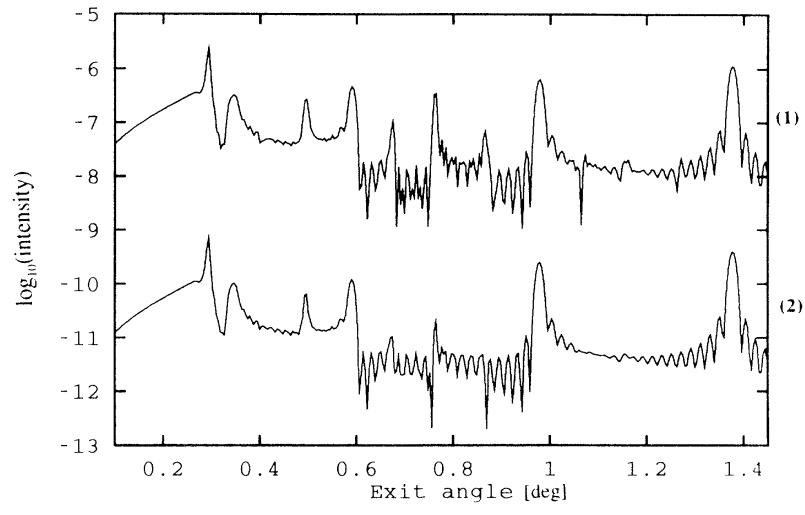

FIG. 14. Influence of rms roughness on the (220) $\alpha_{f}$ scans (relative intensities) for $\alpha_{i}=0.76^{\circ}$, no roughness (1), and $\sigma=1.5$ $\mathrm{nm}(2)$.

\section{Experimental results}

The experimental curves have been fitted by including misorientation and rms roughness. In Fig. 15 we show the best fit of the experimental curves. All peak positions of all curves are well confirmed. There is also good agreement for the intensity ratio of the satellites. Notice that all curves are fitted by the same set of structure parameters. For the mean thickness of the GaAs layers we found $6.65 \pm 0.05 \mathrm{~nm}$, and $14.65 \pm 0.05 \mathrm{~nm}$ for the AlAs barrier. The experimental findings confirm the results of $\mathrm{x}$-ray reflection and conventional symmetrical $\mathrm{x}$-ray diffraction.

The misorientation of the (220) planes has been determined to be $0.03^{\circ} \pm 0.005^{\circ}$. This value has been confirmed by an independent measurement after Ref. 26, applying strong asymmetric coplanar diffraction in reflection and transmission through the edges.

With NSXR measurements we showed evidence of a perpendicular correlation of the roughness of different interfaces in the superlattice. Supported by our NSXR studies we assumed increasing rms roughness for our GID fit. The curves of Fig. 15 are calculated with a starting value at the substrate surface of $\sigma_{\text {sub }}=8.6 \AA$, and a rms roughness of the $j$ th interface of $\sigma_{j}=\sqrt{\sigma_{\text {sub }}^{2}+j \xi^{2}}$ with $\xi=1.6 \AA$.

The experimental curves represent the intrinsic diffraction pattern, convoluted by the intensity distribution within the divergence of the incident beam and by the aperture of the detector.

For a given $\alpha_{i}$, if we want to measure the crystal truncation rod in an $\alpha_{f}$ scan, we have to realize the appropriate lateral divergence of the incident beam $\Delta \theta_{j}$ and a sufficient lateral acceptance of the detector $\Delta \theta_{h}$ according to Eq. (19). Otherwise the detector and the sample have to be rotated to fulfill Eq. (19) for different $\alpha_{f}$ 's.

We performed measurements with fixed sample and PSD positions. Thus the illumination of the TR changes with $\alpha_{i}$. That is the reason for the remaining discrepancies in the measured intensities with respect to the theory, especially for the dramatic decrease in the measured intensity above $1.7^{\circ}$. A banked theoretical $\Delta K$ sa- 
tellite at $\alpha_{i}=\alpha_{f}$ can be avoided by introducing an extinction coefficient dependent on the Bragg-angle deviation for the diffraction. However, the truth lies between the dynamical theory and the distorted-wave approach, since the dynamical theory requires perfect structures. We observed experimental $\left(\alpha_{i}=\alpha_{f}\right)$ peaks, which lay between the results of dynamical theory and the DWBA.
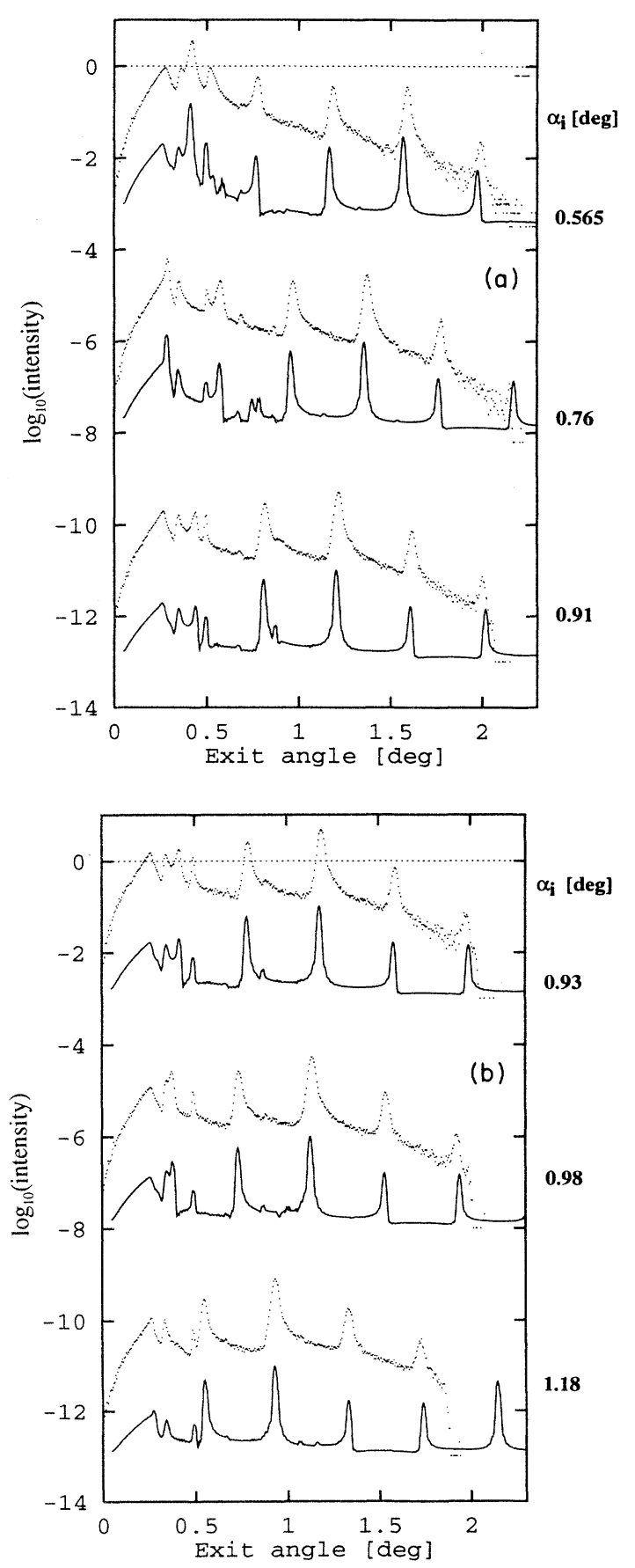

FIG. 15. Best fit of the experimental curves including misorientation and increasing roughness (the curves are shifted progressively along the intensity scale).

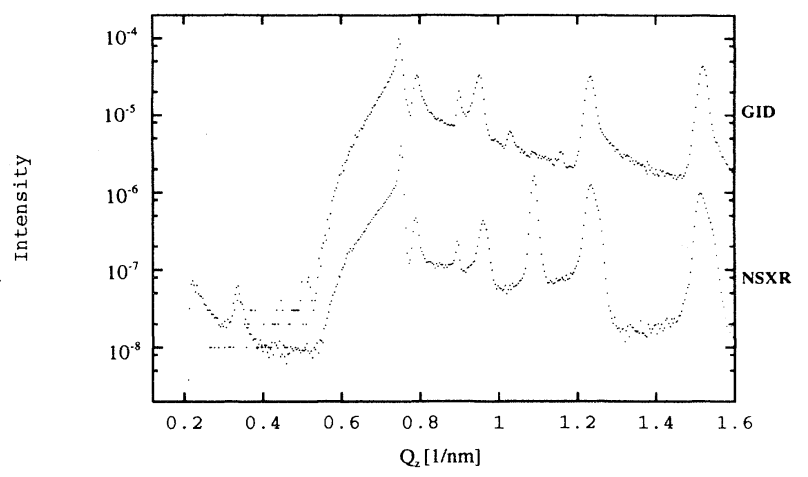

FIG. 16. Comparison of GID and NSXR: measured $Q_{Z}$ scans (relative intensities) at $\alpha_{i}=0.76^{\circ}$.

\section{E. Similarities in the scattering patterns of NSXR and GID}

Finally we want to mention some similarities between the grazing-incidence diffraction pattern and the NSXR pattern for the present case of superlattices with correlated interface roughness. Correlated interface roughness leads to sheets of resonant diffuse scattering (RDS) in the NSXR patterns. These sheets are localized at $q_{z}=2 h \pi / D_{\mathrm{sl}}$, which means they fulfill the same "Bragg" condition as our true satellites. The Bragg-like peaks have also been found in the NSXR patterns. Figure 16 shows two $\alpha_{f}$ scans, the upper one measured in the GID mode, the lower in the $x$-ray-reflection mode. We see the peak positions of the Bragg-like peaks and the primary satellites of both x-ray techniques are well aligned. Also, the agreement of the relative intensities of the satellites is remarkable. In the NSXR pattern the peak of the specular reflected beam occurs at $\alpha_{f}=\alpha_{i}$, where in the GID pattern we find a much weaker $\Delta K$ satellite. Since NSXR has been measured in a coplanar geometry, the expansion

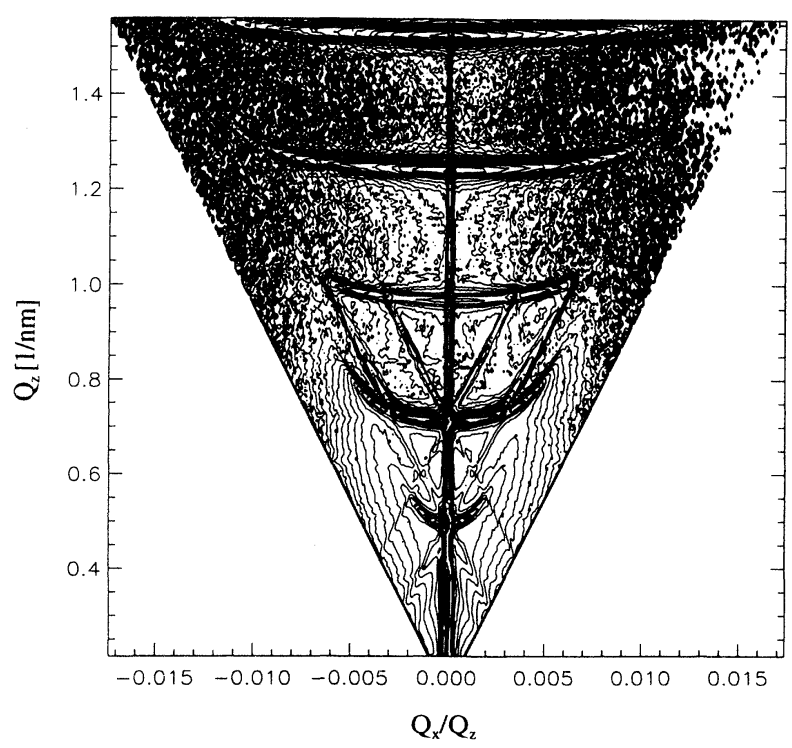

FIG. 17. Contour plot of a NSXR $\left(Q_{Z}, Q_{x} / Q_{z}\right)$ mapping. 
of the $\alpha_{f}$ scan is not limited by the incident lateral acceptances of the monochromator and detector. At angles below the horizon we also observe transmitted orders of resonant diffuse scattering, leaving the sample through the edges.

The similarity of the influences of refraction and Umweganregung due to specular interface reflection can easily be visualized by comparing the $\left(Q_{z}, Q_{\|} / Q_{z}\right)$ mapping of the NSXR intensity, measured from the same sample (Fig. 17) (see Ref. 23), with our $\left(Q_{z}, \alpha_{i}-\alpha_{f}\right)$ mapping of Fig. 10. The RDS-sheets become bent like bananas due to refraction. On the RDS banana-shaped features we observe sharp Bragg-like peaks at the positions where they are crossed by the Bragg-like lines. But notice that the NSXR mapping contains mainly diffuse scattered intensities. Only the SXR truncation rod at $\left(Q_{\|}=0\right)$ represents coherent reflected intensity. Both the RDS banana-shaped features and the Bragg-like lines hit the SXR truncation rod in the SXR Bragg peaks of the superlattice. Unlike the NSXR mapping, the GID $\left(Q_{z}\right.$, $\alpha_{i}-\alpha_{f}$ ) mapping discussed in this paper shows exclusively coherent diffracted intensities. The similarities in both scattering patterns are expressed by the identical unperturbed states of specular reflection, and are further caused by the superlattice periodicity influencing both perturbation potentials.

\section{CONCLUSIONS}

The theoretical treatment of GID based on the distorted-wave approach has been extended to the calculation of the diffracted intensities from epitaxial multilayers. Applying the concept of Umweganregung, we explained the origin of the different features in the diffraction pattern of superlattices.

By the example of a GaAs/AlAs superlattice we have shown that the theory describes details of the diffraction pattern, and have demonstrated the capability of GID for the structural characterization of refraction-indexmodulated superlattices including real-structure effects such as misorientation and graduated interfaces.

\section{ACKNOWLEDGMENTS}

The authors would like to thank F. Morier-Genoud and D. Martin from the Institut de Micro et Optoélectronique, Lausanne (Switzerland) for the sample preparation.
${ }^{1}$ W. C. Marra, P. Eisenberger, and A. Y. Cho, J. Appl. Phys. 50, 6927 (1979).

${ }^{2}$ G. H. Vineyard, Phys. Rev. B 26, 4146 (1982).

${ }^{3}$ A. M. Afanasev and M. K. Melkonyan, Acta Crystallogr. Sec. A 39, 207 (1983).

${ }^{4}$ P. L. Cowan, Phys. Rev. B 32, 5437 (1985).

${ }^{5}$ H. R. Höche, J. Nieber, and O. Brümmer, Acta Crystallogr. Sec. A 42, 585 (1986).

${ }^{6}$ N. Bernhard, E. Burkel, G. Gompper, H. Metzger, J. Peisl, H. Wagner, and G. Wallner, Z. Phys. B 69, 303 (1987).

${ }^{7}$ P. A. Aleksandrov, A. M. Afanasev, A. M. Melkonyan, and S. A. Stepanov, Phys. Status Solidi A 86, 143 (1984).

${ }^{8}$ H. Rhan and U. Pietsch, Z. Phys. B 80, 347 (1990).

${ }^{9}$ S. A. Stepanov, U. Pietsch, and G. T. Baumbach, Z. Phys. B 96, 341 (1995).

${ }^{10}$ T. Baumbach, V. Holy, U. Pietsch, and M. Gailhanou, Physica B 198, 249 (1994).

${ }^{11}$ V. Holy and T. Baumbach, Phys. Rev. B 49, 10668 (1994).

12J. Härtwig, Phys. Status Solidi A 37, 417 (1976).

${ }^{13}$ J. Härtwig, Phys. Status Solidi A 42, 495 (1977).
${ }^{14}$ T. Bedynska, Phys. Status Solidi A 19, 365 (1973).

${ }^{15}$ H. Dosch, Phys. Rev. B 35, 2137 (1987).

${ }^{16}$ U. Pietsch, W. Seifert, J.-O. Fornell, H. Rhan, H. Metzger, S. Rugel, and J. Peisl, Appl. Surf. Sci. 54, 502 (1991).

${ }^{17}$ H. Rhan, U. Pietsch, S. Rugel, H. Metzger, and J. Peisl, J. Appl. Phys. 74, 146 (1993).

${ }^{18}$ D. Rose, U. Pietsch, A. Förster, and T. H. Metzger (unpublished).

${ }^{19}$ U. Pietsch, D. Rose, V. Gottschalch, and H. Rhan, J. Phys. D (to be published).

${ }^{20}$ A. Gonis, Green Functions for Ordered and Disordered Systems (North-Holland, Amsterdam, 1992).

${ }^{21}$ M. Born and E. Wolf, Principles of Optics (Pergamon, London, 1959).

${ }^{22}$ D. K. G. deBoer, Phys. Rev. B 45, 5817 (1994).

${ }^{23}$ V. Holy, T. Baumbach, and M. Bessière, J. Phys. D (to be published).

${ }^{24}$ V. Holy (unpublished).

${ }^{25}$ L. Nevot and P. Croce, Rev. Phys. Appl. 15, 761 (1980).

${ }^{26}$ M. Gailhanou, Appl. Phys. Lett. 63, 458 (1993). 